\title{
Identifying Generative Mechanisms in a Mobile Health (mHealth) Project in Sierra Leone: A Critical Realist Framework for Retroduction
}

\author{
P.J. Wall ${ }^{[0000-0002-5859-4425]}$, Dave Lewis ${ }^{[0000-0002-3503-4644]} \&$ \\ Lucy Hederman ${ }^{\text {[0000-0001-6073-4063] }}$ \\ ADAPT Centre, School of Computer Science and Statistics, Trinity College Dublin, Ireland \\ wallp2@tcd.ie \\ dave.lewis@adaptcentre.ie \\ hederman@tcd.ie
}

\begin{abstract}
This paper examines an ongoing mobile health (mHealth) initiative in Sierra Leone. The objective is to describe the ontological perspective and methodological approach used which relies on critical realism and Archer's morphogenetic approach. Such a critical realist-based approach addresses many of the concerns associated with the interpretivist and positivist philosophical duopoly that has dominated the ICT4D field for many years. The approach allows the mechanisms that determined what caused the events associated with the mHealth case to be hypothesized, thus making clear why this mHealth case has sustained through many challenges.
\end{abstract}

Keywords: ICT4D, mHealth, critical realism, morphogenetic approach

\section{Introduction}

Over the last decade, information and communications technology (ICT) has become more important and ubiquitous across developing countries. This is particularly true of mobile phones which are pervasive in many developing countries. Mobile technologies are also key to meeting the Sustainable Development Goals, and have thus become an integral part of many projects in a variety of fields such as healthcare, emergency management, and food and water security (Masiero 2018). This makes it easy to understand why the number of mHealth implementations around the world has been increasing dramatically (e.g. Purkayastha, Manda, and Sanner 2013, Cameron, Ramaprasad, and Syn 2017, White et al. 2016, Sundin, Callan, and Mehta 2016). However, while mHealth has the potential to transform health services and to increase access to healthcare (e.g. Hurt et al. 2016, Latif et al. 2017, Agarwal et al. 2016, Beratarrechea et al. 2017), the design, implementation and adoption of mHealth in developing countries is beset with wide-ranging challenges and many mHealth projects have been unable to sustain or demonstrate any significant impact at scale (e.g. Chigona, Nyemba, and Metfula 2012, Manda and Msosa 2012, Kahn, Yang, and Kahn 2010, Latif et al. 2017, Sundin, Callan, and Mehta 2016). This is because any mHealth implementation in such environments is bound to be shaped by a variety of 
social, cultural, political, environmental, technological and ideological factors. Failure and underperformance of mHealth projects in these fields has a direct impact on human lives (Masiero 2018), making it important to understand the reasons why so many ICT4D and mHealth projects underperform and fail to scale and sustain.

The ICT4D literature does provide guidance on this and numerous studies have produced insights into what goes wrong (e.g. Avgerou 2007). However, this body of work is dominated by interpretivist and positivist approaches (Gomez 2013, Walsham and Sahay 2006), and each of these paradigms has a number of limitations that constrain ICT4D research (Heeks and Wall 2018). The limitations and weaknesses of interpretivist and positivist approaches have been subject to much debate within the information systems and ICT4D communities over the last decade, and the space constraints imposed by this paper prohibit any in-depth discussion. Suffice it to emphasize that the research paradigm chosen is important as it guides research direction, methodology and conduct. In addition, it determines the way that research questions can be asked and answered and the manner in which findings can be presented (Hughes and Sharrock 2016). Research paradigms therefore determine what we as researchers see and do not see, and what we do and do not do in ICT4D research (Heeks and Wall 2018).

In an attempt to address both the generic and specific concerns associated with the philosophical duopoly that dominates ICT4D research, this paper adopts a "third way” research paradigm: critical realism (Bhaskar 1975, 1979). This philosophical approach is adopted for a variety of reasons, not least of which is the claim that there is a generic ability of critical realism to address issues seen as concerns for ICT4D research (Heeks and Wall 2018). Also, it has been suggested that the field of mHealth in developing countries is better researched by using approaches involving the use of critical theory and critical realism (Lemayian and Omwansa 2013).

This philosophical approach guided the development of a critical realist-based research framework which is applied to an ongoing mHealth case in Bonthe District, Sierra Leone. The aspiration is that the research framework will contribute to providing insights on why this mHealth case sustained through a variety of challenges. The mHealth case is briefly introduced in the following section, with the theoretical framework and methodology presented in section 3. Results are presented in section 4 before the paper concludes with a brief summary.

\section{2 mHealth in Sierra Leone}

In an effort to improve the public health systems, the Ministry of Health in Sierra Leone introduced a policy of integration of voluntary community health workers (CHWs) into the health system in 2012. In an attempt to leverage and aid the work of the CHWs, the Access to Infant and Maternal Health (AIM-Health) Programme (World Vision 2015) was initiated in 2012. AIM-Health contained an mHealth component which commenced as a pilot in Bonthe District, Sierra Leone in January 2013. Nokia Java based mobile phones with an mHealth app preinstalled were given to the CHWs. The mHealth app allowed the CHWs to view which household visits were due, register pregnant women, make emergency referrals to their affiliated health centre, track their own progress, and collect household data for transmission to the health 
facility to support clinical and managerial decision-making (Wall et al. 2013, Vallières et al. 2013).

The mHealth project continued after completion of the pilot phase in April 2014, with ambitious plans in place to scale across Bonthe District and the whole of Sierra Leone by the end of that year. These plans were thrown into chaos when the Ebola virus epidemic struck in May 2014. The epidemic impacted the mHealth project severely with many key people leaving, scarce resources being redeployed, and government restrictions on the movement and association of people. Sierra Leone announced that it was Ebola free in March 2016, but the cost of the epidemic had been immense. According to the World Health Organization (2016) the total death toll in Sierra Leone was 3,955 with 11,308 deaths in total attributed to Ebola across the affected countries in West Africa. It is also estimated that there had been a $23 \%$ decrease in health services delivery in Sierra Leone during that time, with the country losing 7\% of its healthcare workers (Evans, Goldstein, and Popova 2015).

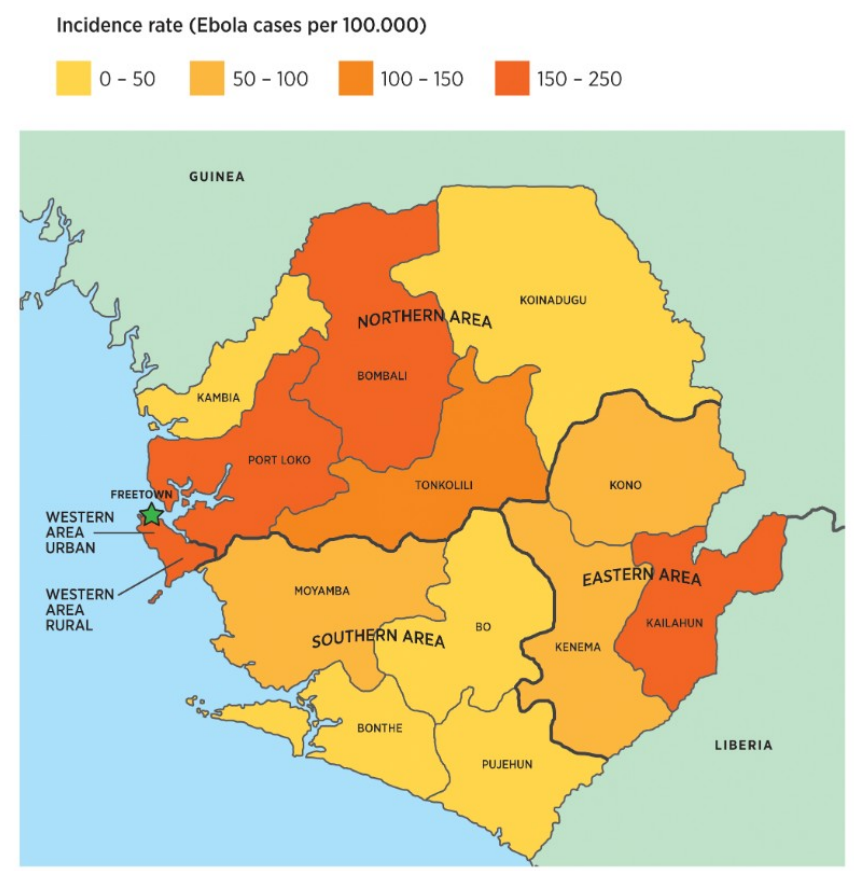

Figure 1: Ebola incidence rates in Sierra Leone (Ribacke et al. 2016)

As can be seen in Figure 1, the incidence of Ebola in Bonthe District was not as high as other areas in Sierra Leone at 0-50 cases per 100,000 people (Ribacke et al. 2016). Despite this, the capacity of the mHealth management team in Bonthe District was greatly reduced during the course of the Ebola epidemic (e.g. the AIM-Health project manager was redeployed, and the digital health M\&E technical specialist left the project), many of the CHWs continued to use the original Nokia mobile phones and mHealth app as part of their work. In addition, a different cohort of CHWs were given Nokia ASHA mobile phones as part of an Ebola community mobilization 
project. This Ebola project trained the CHWs to use the mobile phones to provide information on Ebola to the community, take sick people to the health centre, and report suspected Ebola cases. Although the activity of the CHWs was restricted during this time, data continued to be collected on the original Nokia C2-01 phones that still existed and had a functioning mHealth app installed. This happened even though many of the original phones had been broken, damaged or stolen. Also, the original mHealth app had stopped working at some time during the Ebola crisis as it had ceased to be supported on the Java platform.

\section{Theoretical Framework and Methodology}

This section presents the research question and outlines the theoretical framework adopted. The research adopts a qualitative, longitudinal case study methodology combined with a critical realist ontological perspective. We do not attempt to provide a detailed and comprehensive account of critical realism nor to contribute to the many ontological debates that exist on the relative strengths of various philosophical and ontological approaches to research. Instead, what is presented is an account of critical realism sufficient to provide an understanding of the philosophical and methodological approaches used.

Research questions in critical realist-based research should take a certain form. According to Easton (2010, p. 121) the most fundamental aim of critical realism is explanation, or answers to the question "what caused those events to happen?”. In other words, critical realist-based research questions should be of the form "what caused the events associated with the phenomenon to occur" (Easton 2010, p. 123). Taking this into account, the primary research question asks why the mHealth project in Sierra Leone evolved the way it did, and what were the mechanisms that determined this outcome? An alternative way to ask this question is why did the mHealth project in Sierra Leone turn out the way it did, and what caused the associated events to occur? The question is designed to provide mechanism-based explanation which will indicate how the interaction of different structural, social, cultural, and agency factors have influenced this particular mHealth case.

Put simply, critical realism asserts that general elements of an independent reality exist, but our knowledge of specific structures and mechanisms is limited because of the difficulty of accessing them directly through levels of stratification. This stratification is represented as three nested domains as proposed by Bhaskar (1975). Researchers seek mechanisms, but mechanisms reside in the domain of the Real and are thus independent of human knowledge or our ability to perceive them. The Actual domain contains events which are generated from both exercised and non-exercised mechanisms. The domain of the Empirical contains the events that we as humans are able to experience. 


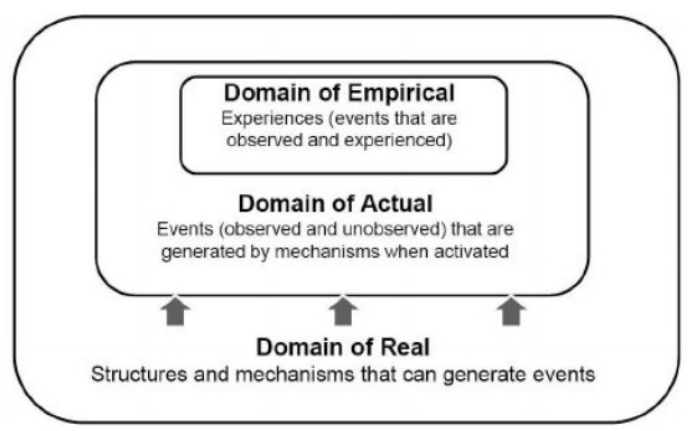

Figure 2: Three overlapping domains of reality in the critical realist ontology (Mingers 2004)

It is important to have a clear understanding of what is meant by "mechanisms" and "events" in this instance. Mechanisms are "causal structures that generate observable events" (Henfridsson and Bygstad 2013, p. 911). An example of an ICT4Drelated mechanism would be an information infrastructure of technology and people in a country that attracts digital service providers, who create new services and thus attract more users, thereby strengthening the information infrastructure and creating a virtuous circle (Bygstad and Munkvold 2011). Events are "specific happenings resulting from causal mechanisms being enacted in some social and physical structure within a particular ... context” (Williams and Karahanna 2013, p. 939). An example of ICT4D-related events might be the appointment of an ICT4D champion, formation of an ICT4D strategy group, or design of an ICT4D app. It is important to mention that critical realism rejects linear notions of causality between mechanisms and events. Instead, it takes an open systems view of the world in which multiple mechanisms intersect, thus creating a "contingent causality" that is context-dependent (Smith 2010).

Retroduction is key to any critical realist-based methodology (Bhaskar 1975), and requires the researcher to take some unexplained phenomenon and propose hypothetical mechanisms that, if they existed, would generate or cause that which is to be explained (Mingers 2004). It is suggested in the literature that the process of applying such retroductive reasoning in an attempt to identify causal mechanisms is challenging (Williams and Karahanna 2013), as well as being difficult, time-consuming and resource-intensive (Reed 2009). However, retroduction is the essential methodological step in all critical realist studies (Mingers, Mutch, and Willcocks 2013) and is required in order to answer our research question.

Our research relies on Margaret Archer's morphogenetic approach (Archer 1995) for retroduction. We do not have the space here to provide detailed explanation of, and discussion on, the morphogenetic approach. Put simply, the morphogenetic approach prioritises agency; it recognises that agents create causation which has the potential to alter structure. This approach is particularly suited to the study of ICT4D as it provides a way to gain better understanding of complex ICT4D contexts (Njihia and Merali 2013). As such, it provides a tractable, comprehensive approach within which we can model and theorise ICT4D change in complex contexts (Njihia and Merali 2013).

For theorization of the mechanisms to occur using the morphogenetic approach, we looked for causal influences and relationships in social structures, interactions and re- 
lationships. Such causal influences and relationships result in either morphogenesis (change) or morphostasis (no change). To achieve this the 4-step methodology as proposed by Raduescu \& Vessey (2008, p. 12) was used as follows:

1. Identification was made of the internal and necessary relations within and between social structure. This was done by asking questions about what needs to be the case, and what needs to be present for $\mathrm{X}$ to be such it is, and not what people think, tell, or believe it is.

2. We then sought causal influences exerted by social structures on social interaction.

3. We then looked for causal relationships between various types of agents.

4. This allowed us to identify how social interaction elaborated upon the composition of social structures by modifying the current internal and necessary structural relations and introducing new ones.

Step 4 identifies morphogenesis which results in transformation. Alternatively, if the social interaction reproduces the existing internal and necessary structural relations then the result of the 4-step methodology is morphostasis which will mean that change does not occur.

The main sources of data used were gathered from semi-structured interviews and focus groups. Over one hundred documents relating to the mHealth case were also analysed. This data was analysed using NVivo software. It is only after the data analysis stage that theoretical reflection can take place. This involves consideration of structural and cultural configuration, and the interactions of the various agents involved and associated outcomes. This research framework allowed us to hypothesize ten separate generative mechanisms. One of these mechanisms is now discussed in detail in the following section.

\section{$4 \quad$ Results, Analysis \& Discussion}

As previously stated, the objective of this research is to identify why the mHealth project evolved the way it did by hypothesizing mechanisms that may have determined this outcome. In other words, we are asking what caused the events associated with this mHealth case to occur. In order to answer this question, we followed the research framework as presented in Figure 3 and outlined in the previous section.

Firstly, data was collected by semi-structured interview, focus group discussion, observation and document analysis. This data was then used to construct a factual case study description and a detailed chronological account of events. These documents give rise to the identification of discrete time periods called morphogenetic/ morphostatic $(\mathrm{M} / \mathrm{M})$ cycles which are identified empirically from the factual case study description and the chronological account of events. Three distinct M/M cycles were identified as follows:

1. The mHealth planning and pilot cycle (2012 to April 2014). This cycle includes planning for the implementation of mHealth as part of the AIM-Health project. It also includes the mHealth pilot itself up to May 2014, where the CHWs were using the original Nokia C2-01 mobile phones and mHealth app. 
2. Ebola virus outbreak cycle (May 2014 to March 2016). This cycle includes the loss of key staff from the mHealth management team in Bonthe District, and the retraining of the CHWs as part of the Ebola community mobilization project. It also includes the CHWs use of both the original mobile phones and the Nokia ASHA phones during this time.

3. Post-Ebola cycle (March 2016 to 2018). This cycle includes the use of the mobile phones by the CHWs in the post-Ebola period.

Each of the distinct M/M cycles were analysed using the 4-step methodology as proposed by Raduescu \& Vessey (2008, p. 12) to produce analytical histories of emergence. These analytical histories of emergence then formed the basis for retroduction. This allowed us to retroduce a total of ten generative mechanisms. Examples of the mechanisms retroduced are the communications and technological infrastructure built around the mHealth project mechanism; the self-reinforcing adoption of mHealth mechanism (which includes mHealth policy, good governance and supervision structures, inclusivity, and participatory design and decision making); the establishment of

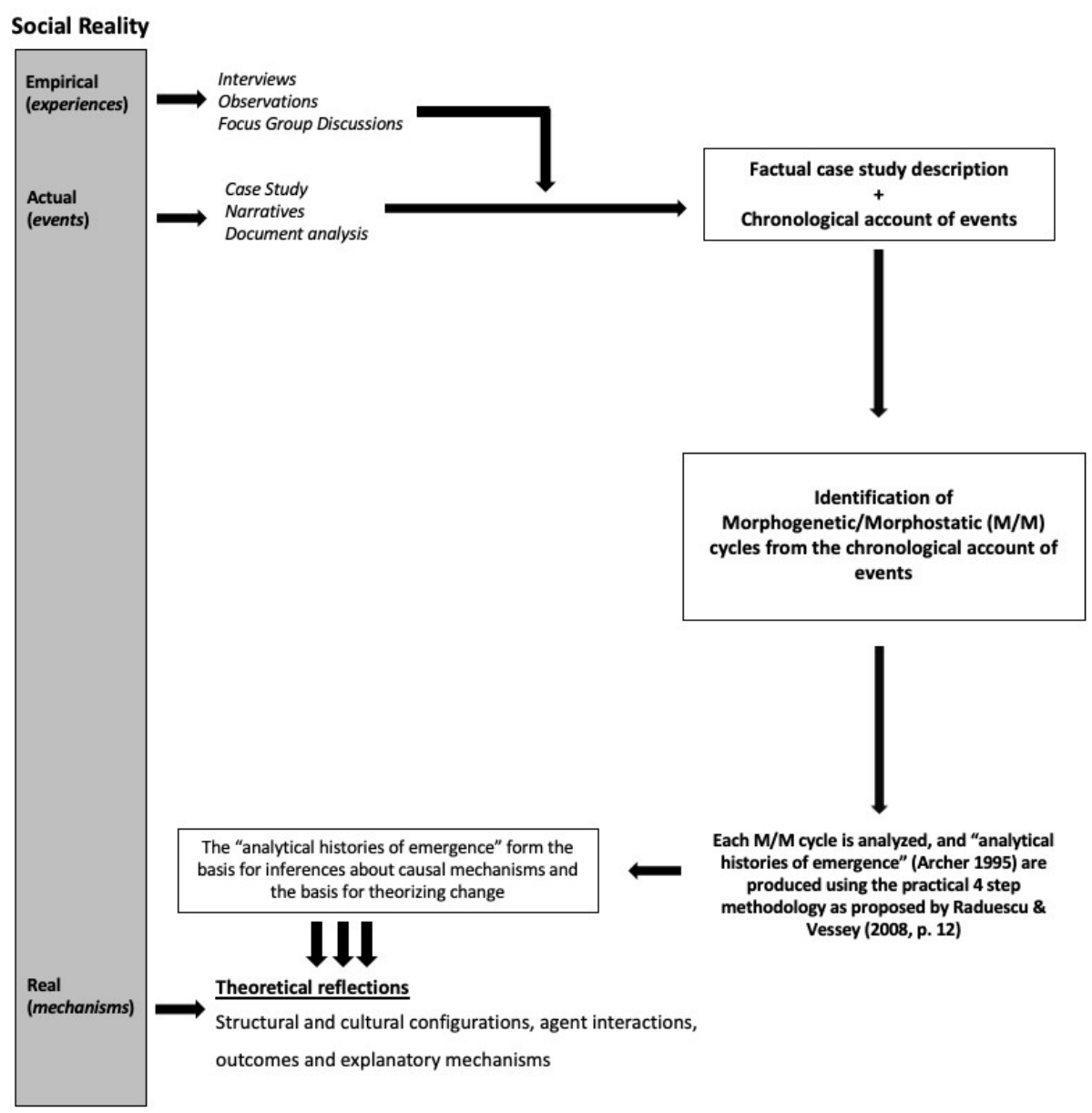


Figure 3: Diagrammatic representation of the theoretical framework adopted for this research.

the CHW hub and the eHealth hub by the Ministry of Health in Sierra Leone; and the monitoring and reporting capabilities of the mobile phones and mHealth app.

The space restrictions imposed by this paper allow us to discuss only one of the mechanisms in any detail. The mechanism now discussed is the communications and technological infrastructure built around the mHealth project mechanism. This infrastructure consists of technology, people and monitoring and reporting structures.

An important component of this mechanism is the two-way communication structures that operate between the CHWs at community level, the district health management team at district level, and the Ministry of Health at national level. CHWs attached to each of the health centers in Bonthe District have monthly meetings where they raise issues that they see as important, and they can also expect to receive feedback on their performance. These monthly meetings may be attended by a variety of stakeholders including the health staff, various NGO representatives, local politicians and citizen representatives. These meetings will then be reported back to the district health management team in Bonthe District. This allows the concerns and performance of the CHWs to be communicated directly to the district health management team. A report from this meeting is then given directly to the Ministry of Health in Freetown. This communication system also works the opposite way, i.e. from national level back to district level and then to the CHWs at community level.

We suggest that this mechanism contributes to attracting and maintaining CHWs for many reasons. This is because the CHWs know they will be monitored and trained in an efficient manner, and as a result they can do their jobs more effectively. They also have access to an effective channel for communicating their concerns and ideas. This allows problems to be addressed and the work of the CHW to be completed more effectively. This has the effect of strengthening the health systems as well as making the job of the CHW a more desirable one.

\section{Summary}

It is both challenging and ambitious to attempt any level of understanding of mHealth in a severely resource constrained environment such as Sierra Leone. This research adopts a critical realist perspective to identify why the mHealth project evolved the way it did by hypothesizing mechanisms that may have determined the outcome of the project. The hypothesized mechanisms explain how the interaction of different structural, cultural and agency factors have influenced the project. All mechanisms hypothesized by this work are equally important and go towards explaining why the mHealth project evolved the way it did. Our belief is that such mechanism-based explanation allows a theoretically informed and empirically rich account of how context and mechanism interact in this specific case. 


\section{References}

Agarwal, Smisha, Amnesty E LeFevre, Jaime Lee, Kelly L’Engle, Garrett Mehl, Chaitali Sinha, and Alain Labrique. 2016. "Guidelines for reporting of health interventions using mobile phones: mobile health (mHealth) evidence reporting and assessment (mERA) checklist." Bmj 352:i1174.

Archer, M.S. 1995. Realist Social Theory: The Morphogenetic Approach. Cambridge, UK: Cambridge University Press.

Avgerou, C. 2007. "Title." LSE Working papers series.

Beratarrechea, Andrea, Daniela Moyano, Vilma Irazola, and Adolfo Rubinstein. 2017. "mHealth Interventions to Counter Noncommunicable Diseases in developing countries: still an Uncertain Promise." Cardiology clinics 35 (1):13-30.

Bhaskar, R. 1975. A Realist Theory of Science. Hassocks, England: Harvester Press.

Bhaskar, R. 1979. The Possibility of Naturalism. Brighton, UK: Harvester.

Bygstad, B., and B.E. Munkvold. 2011. "In Search of Mechanisms. Conducting a Critical Realist Data Analysis." ICIS.

Cameron, Joshua D, Arkalgud Ramaprasad, and Thant Syn. 2017. "An ontology of and roadmap for mHealth research." International journal of medical informatics 100:16-25.

Chigona, W., M. Nyemba, and A. Metfula. 2012. "A review on mHealth research in developing countries." The Journal of Community Informatics 9 (2).

Easton, Geoff. 2010. "Critical realism in case study research." Industrial Marketing Management 39 (1):118-128.

Evans, David K, Markus Goldstein, and Anna Popova. 2015. "Health-care worker mortality and the legacy of the Ebola epidemic." The Lancet Global Health 3 (8):e439-e440. 
Gomez, R. 2013. "The Changing Field of ICTD: Growth and maturation of the field, 2000-2010." The Electronic Journal of Information Systems in Developing Countries 58.

Heeks, R. , and P.J. Wall. 2018. "Critical realism and ICT4D research." The Electronic Journal of Information Systems in Developing Countries 84 (6).

Henfridsson, O., and B. Bygstad. 2013. "The generative mechanisms of digital infrastructure evolution." MIS Quarterly 37 (3).

Hughes, John A, and Wes W Sharrock. 2016. The philosophy of social research: Routledge.

Hurt, Kathryn, Rebekah J Walker, Jennifer A Campbell, and Leonard E Egede. 2016. "mHealth interventions in low and middle-income countries: a systematic review." Global journal of health science 8 (9):183.

Kahn, J.G., J.S. Yang, and J.S. Kahn. 2010. "'Mobile’health needs and opportunities in developing countries." Health Affairs 29 (2):252-258.

Latif, Siddique, Rajib Rana, Junaid Qadir, Anwaar Ali, Muhammad Ali Imran, and Muhammad Shahzad Younis. 2017. "Mobile health in the developing world: Review of literature and lessons from a case study." IEEE Access 5:1154011556.

Lemayian, N.M., and T.K. Omwansa. 2013. "Towards a Critical Realist Epistemology for M-Health Research." International Journal of Information and Communication Technology Research 3 (6):205-210.

Manda, T.D., and Y. Msosa. 2012. "Socio-technical Arrangements for mHealth: Extending the Mobile Device Use and Adoption Framework." In eInfrastructure and e-Services for Developing Countries, edited by Radu Popescu-Zeletin, Karl Jonas, IdrisA Rai, Roch Glitho and Adolfo Villafiorita, 208-217. Springer Berlin Heidelberg.

Masiero, S. 2018. "Explaining trust in large biometric infrastructures: a critical realist case study of India's Aadhaar project " The Electronic Journal of Information Systems in Developing Countries 84 (6).

Mingers, J. 2004. "Real-izing information systems: critical realism as an underpinning philosophy for information systems." Information and organization 14 (2):87-103.

Mingers, J., A. Mutch, and L. Willcocks. 2013. "Critical Realism in Information Systems Research." MIS Quarterly 37 (3):795-802.

Njihia, J.M., and Y. Merali. 2013. "The broader context for ICT4D projects: a morphogenetic analysis." MIS Quarterly 37 (3).

Purkayastha, S., T.D. Manda, and T.A. Sanner. 2013. "A Post-development Perspective on mHealth -- An Implementation Initiative in Malawi." 2013 46th Hawaii International Conference on System Sciences (HICSS).

Raduescu, C., and I. Vessey. 2008. "Causality in Critical Realist Research: An Analysis of Three Explanatory Frameworks." Proceedings of The International Association for Critical Realism Annual Conference 2008, London, UK: 11-13 July 2008, 11-13 July 2008.

Reed, M. 2009. "Critical realism: Philosophy, method, or philosophy in search of a method?" In The SAGE handbook of organizational research methods, edited by David Buchanan \& Alan Bryman, 430-448. London: SAGE. 
Ribacke, Kim J Brolin, Alex J van Duinen, Helena Nordenstedt, Jonas Höijer, Ragnhild Molnes, Torunn Wigum Froseth, AP Koroma, Elisabeth Darj, Håkon Angel Bolkan, and AnnaMia Ekström. 2016. "The impact of the West Africa Ebola outbreak on obstetric health care in Sierra Leone." PLOS ONE 11 (2):e0150080.

Smith, M.L. 2010. "Limitations to building institutional trustworthiness through egovernment: a comparative study of two e-services in Chile." Journal of Information Technology 26 (1):78-93.

Sundin, Phillip, Jonathan Callan, and Khanjan Mehta. 2016. "Why do entrepreneurial mHealth ventures in the developing world fail to scale?" Journal of medical engineering \& technology 40 (7-8):444-457.

Vallières, F., E. McAuliffe, A. Bangura, A. Trye, and P.J. Wall. 2013. "The Implications of mHealth for Human Health Resources in Bonthe District, Sierra Leone." eChallenges e-2013 Conference Proceedings, Dublin, Ireland.

Wall, P.J., F. Vallières, D. Lewis, L. Hederman, and J. Musa. 2013. "A SocioTechnical Approach to the Implementation of mHealth in Sierra Leone: A Theoretical Perspective." eChallenges e-2013 Conference Proceedings, Dublin, Ireland.

Walsham, G., and S. Sahay. 2006. "Research on information systems in developing countries: current landscapes and future prospects." Information Technology for Development 12 (1):7 - 24.

White, Alice, Deborah SK Thomas, Nnamdi Ezeanochie, and Sheana Bull. 2016. "Health worker mHealth utilization: a systematic review." Computers, informatics, nursing: CIN 34 (5):206.

Williams, C.K., and E. Karahanna. 2013. "Causal Explanation in the Coordinating Process: A Critical Realist Case Study of Federated IT Governance Structures." Mis Quarterly 37 (3):933-964.

World Health Organization (WHO). 2016. Ebola Situation Report. Geneva: World Health Organization.

World Vision. 2015. Access Infant and Maternal Health (AIM-Health) - End Line Programme Evaluation Report. Dublin, Ireland: Irish Aid. 